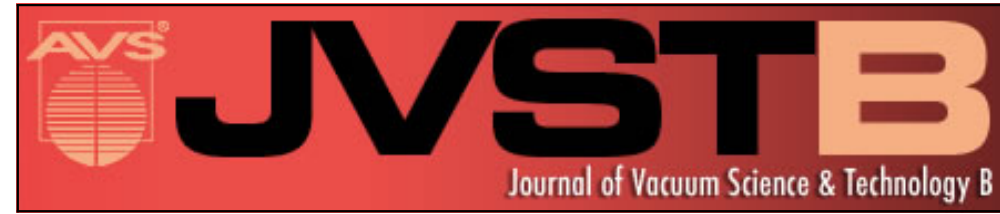

\title{
Combination of fluorescence in situ hybridization and scanning force microscopy for the ultrastructural characterization of defined chromatin regions
}

W. Fritzsche, L. Takács, G. Vereb, J. Schlammadinger, and T. M. Jovin

Citation: Journal of Vacuum Science \& Technology B 14, 1399 (1996); doi: 10.1116/1.589107

View online: http://dx.doi.org/10.1116/1.589107

View Table of Contents: http://scitation.aip.org/content/avs/journal/jvstb/14/2?ver=pdfcov

Published by the AVS: Science \& Technology of Materials, Interfaces, and Processing

\section{Articles you may be interested in}

Combination of magnetic force microscopy with in situ magnetoresistance measurements

J. Appl. Phys. 92, 1014 (2002); 10.1063/1.1485108

Ultrastructural characterization of colloidal metal films for bioanalytical applications by scanning force microscopy J. Vac. Sci. Technol. A 14, 1766 (1996); 10.1116/1.580334

Nanostructural characterization of thin-film transistors using a combination of scanning force microscopy and transmission electron microscopy

J. Vac. Sci. Technol. A 14, 1714 (1996); 10.1116/1.580325

Reconstruction of ribosomal subunits and rDNA chromatin imaged by scanning force microscopy

J. Vac. Sci. Technol. B 14, 1405 (1996); 10.1116/1.589108

Combined scanning tunneling and force microscopy

J. Vac. Sci. Technol. B 12, 1677 (1994); 10.1116/1.587261

\section{PIDEN}

Contact Hiden Analytical for further details: w www.HidenAnalytical.com E info@hiden.co.uk CLICK TO VIEW our product catalogue

\section{Instruments for Advanced Science}

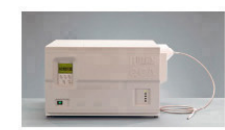

Gas Analysis

dynamic measurement of reaction gas streams , catalysis and thermal analysis

molecular beam studies

fermentation, environmental and ecological studies

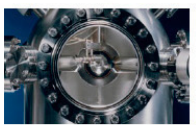

Surface Science UHVTPD SIMS

end point detection in ion beam etch elemental imaging - surface mapping

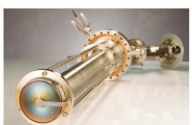

Plasma Diagnostics ; plasma source characterization etch and deposition process reaction kinetic studies , analysis of neutral and radical species

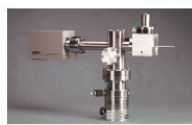

Vacuum Analysis - partial pressure measurement and control of process gases reactive sputter process control vacuum diagnostics vacuum coating process monitoring 


\title{
Combination of fluorescence in situ hybridization and scanning force microscopy for the ultrastructural characterization of defined chromatin regions
}

\author{
W. Fritzsche ${ }^{\text {a) }}$ \\ Department of Molecular Biology, Max Planck Institute for Biophysical Chemistry, P.O. Box 2841, \\ 37018 Göttingen, Germany \\ L. Takács \\ Department of Ophthalmology, University Medical School of Debrecen, P.O. Box 30, 4012 Debrecen, \\ Hungary and Dept. of Molecular Biology, Max Planck Institute for Biophysical Chemistry, \\ P.O. Box 2841, 37018 Göttingen, Germany \\ G. Vereb \\ Department of Molecular Biology, Max Planck Institute for Biophysical Chemistry, P.O. Box 2841, \\ 37018 Göttingen, Germany \\ J. Schlammadinger \\ Department of Biology, University Medical School of Debrecen, P.O. Box 2, 4012 Debrecen, Hungary \\ T. M. Jovin ${ }^{\text {b) }}$ \\ Department of Molecular Biology, Max Planck Institute for Biophysical Chemistry, P.O. Box 2841, \\ 37018 Göttingen, Germany
}

(Received 25 July 1995; accepted 15 December 1995)

\begin{abstract}
Although the internal arrangement of interphase chromatin is still a matter of conjecture, there exists a large body of evidence for the compartmentalization of chromosomal domains. A study based on combined scanning force and optical microscopy of supramolecular chromatin spreads produced by isotonic lysis of cells suspended in phosphate-buffered saline has been conducted. The ultrastructure of fluorescent labeled chromosomes was resolved with the topographical contrast provided by the scanning force microscope. Fluorescence in situ hybridization was used to label specific DNA sequences. The location of different pericentromeric chromosome regions was determined by fluorescence microscopy and correlated with scanning force microscope topography. Using a single DNA probe, discrimination between labeled chromosome pairs of an aneuploid cell was possible, based on the different intensities of fluorescence signals. The results show that the in situ hybridization technique with fluorescence labeling is compatible with scanning force microscopy. The combination of these methods can be used for the specific identification and lateral localization of DNA sequences in spread chromatin, opening the possibility for the ultrastructural characterization of defined genes in the scanning force microscope. (C) 1996 American Vacuum Society.
\end{abstract}

\section{INTRODUCTION}

The length of human genomic extended DNA is about 1.8 $\mathrm{m}$. The DNA in association with histone and nonhistone proteins constitutes the system of chromatin fibers and is packaged in an interphase cell nucleus $\sim 5 \mu \mathrm{m}$ in diameter. In the course of the cell cycle, the chromatin undergoes further compaction, culminating in the highly condensed metaphase chromosomes. The fundamental morphological feature of chromatin is the nucleosomal chain, constituted of DNA and histone proteins and establishing the typical "beads-on-astring" conformation in the electron microscope ${ }^{1}$ or scanning force microscope (SFM). ${ }^{2,3}$ This nucleosome chain can be further compacted into a $\sim 30 \mathrm{~nm}$ fiber under low ionic strength conditions. ${ }^{4,5}$ Chromatin fibers $100 \mathrm{~nm}$ in width have also been shown in G1 interphase nuclei. ${ }^{6}$ The mecha-

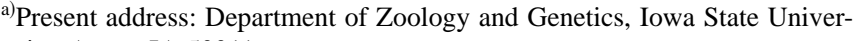
sity, Ames, IA 50011

${ }^{b)}$ Author to whom correspondence should be addressed. nism of further compaction of chromatin fibers in interphase nuclei and the transition from the relaxed interphase chromatin to the very compact metaphase chromosomes are poorly understood. There is evidence for compartmentalization of chromosomal domains in interphase, ${ }^{7}$ and some information has been derived from statistical analysis of metaphase spreads based on the assumption that the metaphase chromosome distribution should reflect, at least to some extent, the topology of chromosomes inherited from the preceding interphase. $^{8}$ Studies of sectioned metaphase cells ${ }^{9}$ and metaphase spreads ${ }^{10}$ show that larger chromosomes have a more peripheral position.

$\mathrm{SFM}^{11}$ is a new high-resolution technique with an increasing number of applications in structural biology, ${ }^{12-15}$ including studies of chromatin (for a review see Ref. 16). Investigations of isolated chromatin fibers ${ }^{17-19}$ and spread nuclei ${ }^{20}$ have been reported. The use of spreading techniques that preserve, at least to some extent, the spatial relations of 
(a)

(b)

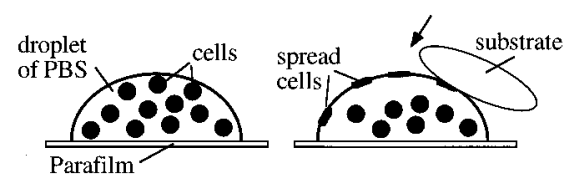

(C)

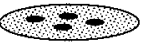

FIG. 1. Sample preparation procedure. During isotonic lysis the cells are suspended in an isotonic buffer (PBS) and a $60 \mu$ droplet of cell suspension is deposited onto a Parafilm layer (a). Some cells spread out on the surface of the PBS droplet. The forces involved in this process are still unknown, but it is likely that surface tension forces play an important role in the spreading of the cells. After 20 min the spread cells can be harvested from the droplets by briefly touching a suitable substrate (glass coverslip in this case) to the surface (b); the arrow indicates the direction of movement. Most of the cytoplasmic components of the spread cells are lost during the procedure. The spread nuclear material (the chromatin) forms "plaques" on the substrate and, after washing and fixation, is suitable for further investigations (c).

neighboring chromatin sites in the interphase nucleus permits the investigation of chromatin at a spatial resolution otherwise hindered by the tight packaging of the genetic material. One of these techniques, isotonic lysis, ${ }^{21}$ leads to spreading of interphase chromatin without applying detergent or resorting to hypotonic conditions.

The investigation of single chromosomes during interphase is hampered by the decondensation of chromatin such that identification according to structural features is impossible; however, a labeling of specific DNA sequences in chromatin can be achieved by in situ hybridization. There are several ways of visualizing the labeled DNA sequences, one of them being the attachment of fluorescent dyes to the probe, designated as fluorescence in situ hybridization (FISH). By using labeled chromosome specific libraries, FISH allows the detection of chromatin material corresponding to single chromosomes in interphase nuclei. ${ }^{22}$

In situ hybridization combined with cytochemical techniques has been used for SFM investigations of metaphase chromosomes. ${ }^{23,24}$ An enzyme-catalyzed growth of amorphous material at the hybridization site was used for detection. The resolution was limited by the detection method and the extreme condensation of the chromatin in the metaphase chromosome. The use of fluorescence-based detection applied to spread chromatin should result in a considerable increase in the lateral resolution of the hybridization site. The application of simultaneous fluorescence and SFM investigations of the same specimen of spread chromatin was demonstrated previously. ${ }^{20}$ In this study we demonstrate the compatibility of FISH and SFM, a combined tool ideally suited for ultrastructural investigations of chromatin related to specified DNA sequences.

\section{EXPERIMENTAL PROCEDURES}

JY human B lymphoblastoid cells ${ }^{25}$ were grown in RPMI1640 medium supplemented with $10 \%$ fetal calf serum (FCS) in a humidified $\mathrm{CO}_{2}$ incubator at $37^{\circ} \mathrm{C}$. The cells were spread according to Schlammadinger. ${ }^{21}$ The cell suspension was washed twice in PBS and resuspended to $10^{6}$
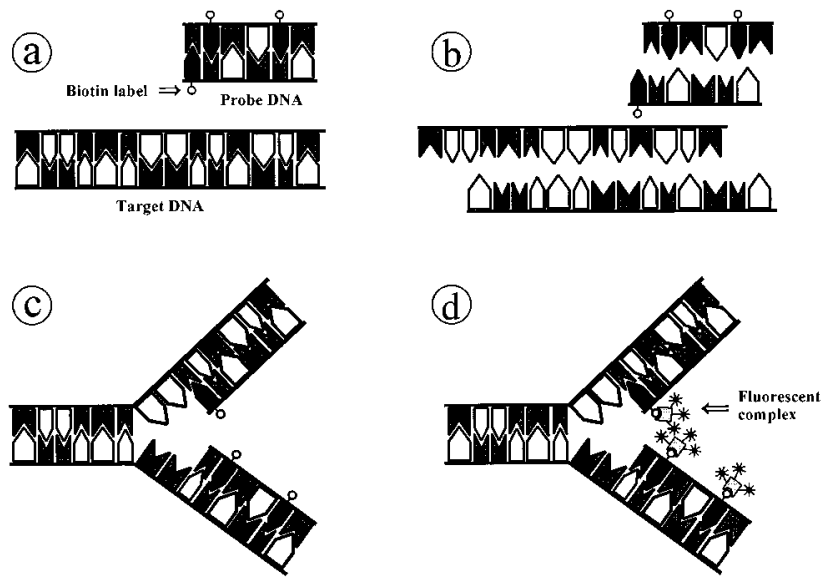

FIG. 2. Fluorescence in situ hybridization (FISH). This method is suitable for labeling specific DNA sequences in the cells using haptens (usually biotin or digoxigenin) attached to probes complementary to the sequences to be detected. During the procedure the labeled probe DNA is added in excess to the target (cellular) DNA (a). The DNA is then denatured, usually by heating (b). In the following step, the labeled DNA probe and the sample DNA are cooled together. DNA renatures, and the labeled probe hybridizes to the complementary sites of the sample DNA (c). The hybridization signal is detected by fluorophore conjugated antibodies or proteins that specifically recognize the hapten bound to the probe (d). The fluorescent signal gained in this way can be further amplified using fluorophore conjugated second and third antibodies.

cells/ml. Droplets of $60 \mu \mathrm{l}$ were deposited on Parafilm. After incubation for $20 \mathrm{~min}$, each droplet was touched with a thoroughly cleaned glass coverslip (Fig. 1). The spreads adhering to the coverslips were fixed for $2 \mathrm{~h}$ in $2 \%$ paraformaldehyde (PFA) in phosphate buffered saline (PBS), washed in PBS, washed in distilled water, and air dried.

FISH of air-dried samples was performed according to Speleman et al. (Fig. 2). ${ }^{26}$ We used biotinylated DNA probes pUC1.77 and L1.26, a kind gift from Dr. F. Speleman (Dept. of Medical Genetics, University Hospital of Ghent, Belgium). The probe pUC1.77 binds to a $\sim 10^{5}$ base pair long repetitive sequence in the pericentromeric heterochromatin of chromosome $1 \mathrm{q},{ }^{27}$ while the L1.26 recognizes alphoid repetitive sequences in the pericentromeric heterochromatin of chromosomes 13 and $21 .^{28}$ The rehydrated samples were digested with RNaase A for $60 \mathrm{~min}$, then with pepsin for 30 min at $37{ }^{\circ} \mathrm{C}$. After $5 \mathrm{~min}$ postfixation at room temperature in $4 \%$ PFA and $0.5 \% \mathrm{MgCl}_{2}$, the samples were dehydrated in ethanol series and air dried. The chromatin samples and the probe DNA were denatured together in $60 \%$ formamide/ $2 \times \mathrm{SSC}$ at $90{ }^{\circ} \mathrm{C}$ for $5 \mathrm{~min}$, and hybridized overnight at $37^{\circ} \mathrm{C}$. Probe detection was with a first step of fluoresceinisothiocyanate (FITC) labeled Neutralite-Avidin followed by an amplification step using biotinylated goat anti-avidin and Neutralite-Avidin-FITC. The DNA was labeled using 4', 6-diamidino-2' phenylindole (DAPI) as described earlier. ${ }^{20}$

Scanning force microscopy was performed with a NanoScope III contact SFM (Digital Instruments, Santa Barbara, CA), operating in the topographic (isoforce) mode, and using a J-tube scanner with a $130 \times 130 \times 5(x, y, z) \mu \mathrm{m}$ maximum scan range. The calibration is discussed in detail elsewhere. ${ }^{29}$ Si tips with a radius of $\sim 5 \mathrm{~nm}$ and a cantilever force con- 

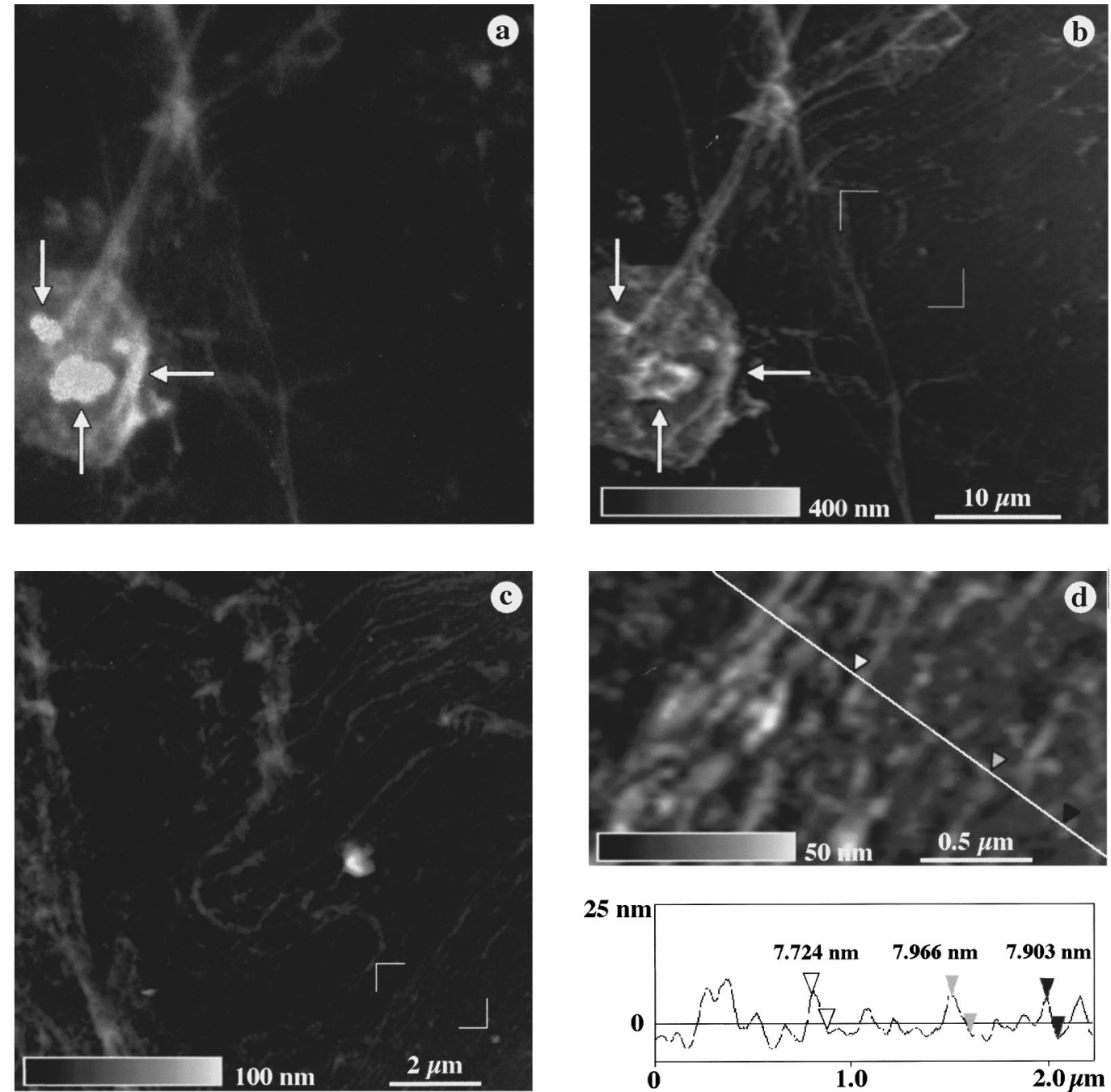

FIG. 3. Fluorescence microscopic images of spread chromatin. Overlay of the signal originating from DAPI staining of nucleic acids and high-intensity spots marked with arrows, representing the FITC fluorescence from the same area (a). The DAPI image revealed a distinct plaque containing the chromatin material of a single nucleus. Some chromatin fibers radiated outward from the plaque. The FITC label localized the hybridization probe L1.26 inside the chromatin plaques (arrows). The same topographic pattern was observed by SFM (b). The protein complexes built up on the hybridization sites are also revealed. At higher magnifications chromatin fibers of different sizes (c) down to fine threads of 7.7-8 nm height and 40-60 nm width (d) were observed. The height vs distance diagram (linescan) was extracted from the section of the image marked by the diagonal line. The arrowheads point to the sites at which the filament dimensions were measured. The location of the higher-resolution images in (c) and (d) are marked at their corners in frames (b) and (c), respectively. Gray-scale bars code height information, the scale bars show lateral dimensions.

stant of $\sim 0.03 \mathrm{~N} / \mathrm{m}$ (Ultralever, Park Scientific Instruments, Sunnyvale, CA) were used. Images of $512 \times 512$ pixels were obtained at line scan rates of $5-7 \mathrm{~Hz}$. Image processing and analysis were carried out using the NanoScope software. Estimation of the lateral dimensions of topographic features was done at half maximum height. ${ }^{3}$

Fluorescence imaging was with a Zeiss Axioplan epifluorescence microscope equipped with a Photometrics Series 200 cooled slow scan charge-coupled-device (CCD) camera system incorporating a Kodak KAF 1400 sensor (Photometrics, Tucson, AZ), and interfaced to a Macintosh Quadra 800 computer (Apple Computer, Cupertino, CA). For orienting the samples, images of DAPI-stained dry spreads were taken at low magnification with a Plan-Neofluar $20 \times$ N.A. 0.5 objective. After SFM investigation of selected regions the samples were rehydrated, washed, restained with DAPI, and mounted in Mowiol (Hoechst). Images of DAPI and FITC fluorescence were taken using the appropriate Zeiss filter sets and a Plan-Neofluar $100 \times$ N.A. 1.3 objective. Digitized images were processed and aligned to their SFM counterpart using the program NIH-Image.

\section{RESULTS AND DISCUSSION}

During isotonic lysis the chromatin of the cells suspended in PBS droplets spreads on the surface of the buffer. The spread chromatin can be transferred to a glass coverslip by touching it to the surface of the droplets (Fig. 1). According to our results the chromatin spreads prepared in this way are 

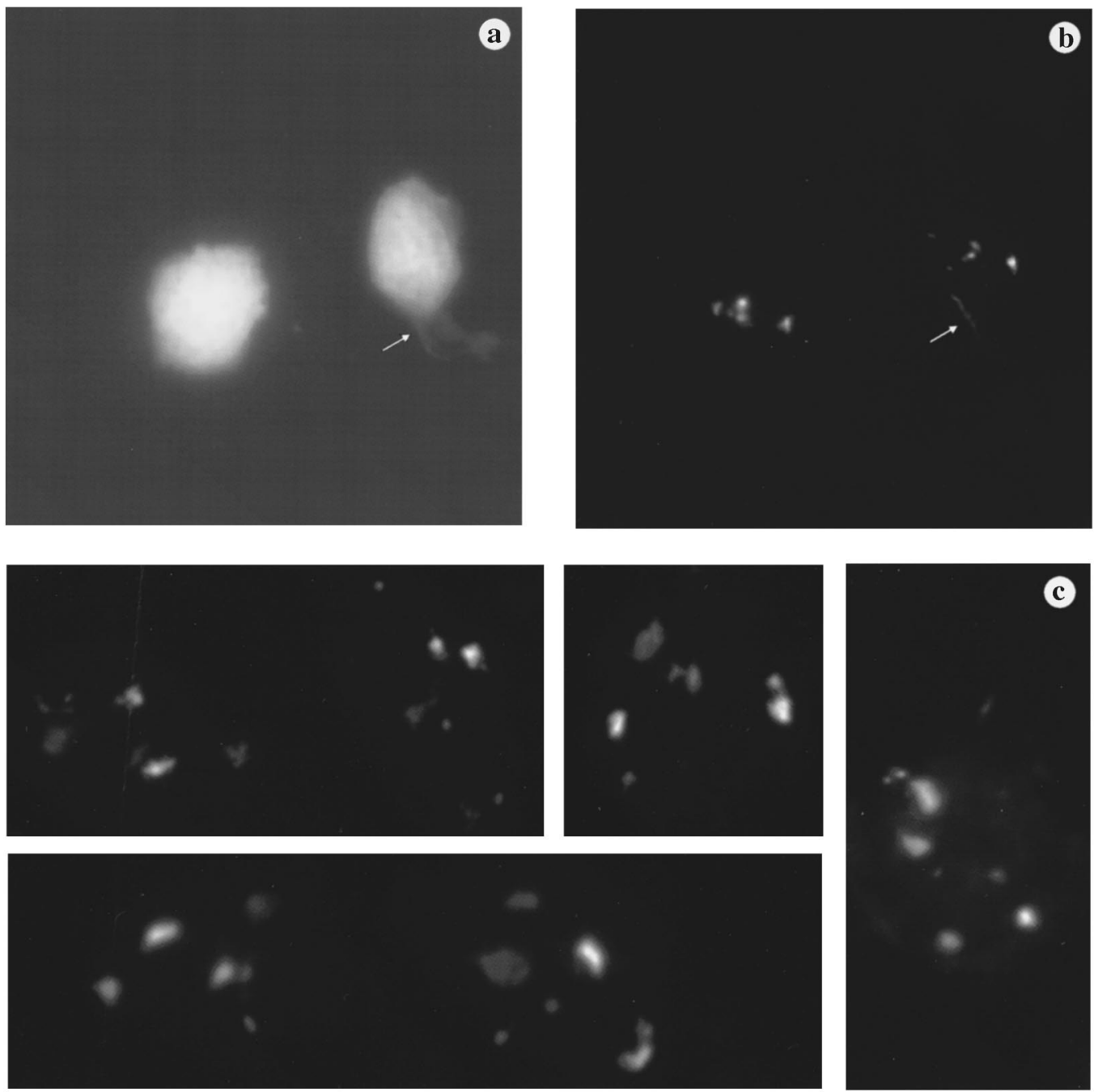

FIG. 4. DAPI and FISH images of spread chromatin. The FISH signals correspond to hybridization with the pUC1.77 probe. The DAPI image (a) shows two chromatin plaques, and a bundle of chromatin fibers extending out from one of the plaques (arrow). Owing to large differences in intensity the log of intensity was displayed on a gray scale to better visualize both strong fluorescence from compact regions and weak signals from the spread fibers (image size $75 \mu \mathrm{m}$ ). FISH signals from the same area are shown in (b). Six to eight spots of different size and intensity were located in each DAPI stained region. One of these hybridization signals was located on the extended chromatin fibers and showed a length of $\sim 5 \mu \mathrm{m}$ (arrow) instead of the more common spots. FISH signals from several spread nuclei are presented in panel (c). The 6-8 spots visible in each chromatin spread could be paired based upon distinctive sizes and intensities.

stable enough to allow subsequent in situ hybridization (Fig. 2) and scanning force microscopic investigations of the same specimen.

Figure 3 shows fluorescent and scanning force micrographs of spread chromatin samples. The L1.26 probe was used for the in situ hybridization and visualized thereafter with FITC fluorescence. The DNA content of the chromatin material was detected with DAPI. The two images were overlaid in order to demonstrate the localization of the FISH signals relative to the DNA content. Fluorescent images like those of Fig. 3(a) showed that the chromatin material from single cell spreads formed distinct plaques with some chromatin fibers radiating outward. The signals of the hybridization probe were surrounded by the chromatin, demonstrating the conserved integrity of the spread nuclear content. Neither disruption nor partition of the chromatin of intact nuclei was observed.

The density of the plaques was $\sim 100 / \mathrm{mm}^{2}$. The shapes were mostly round, with diameters of $\sim 10-20 \mu \mathrm{m}$. The fluorescent hybridization signals were located in distinct regions of the spread-chromatin plaques (arrows). The SFM image [Fig. 3(b)] revealed the same pattern as seen in the fluorescent microscope. This suggests that the topographic contrast is built up by DNA-containing material, i.e., chromatin. Plaque heights of up to $500 \mathrm{~nm}$ were measured. A height difference of 150-200 $\mathrm{nm}$ between the signalcontaining regions and the surrounding chromatin material was observed. The site to which the L1.26 probe hybridized comprises alphoid repetitive sequences located in close proximity of the nucleolus organizing region on the acrocentric 

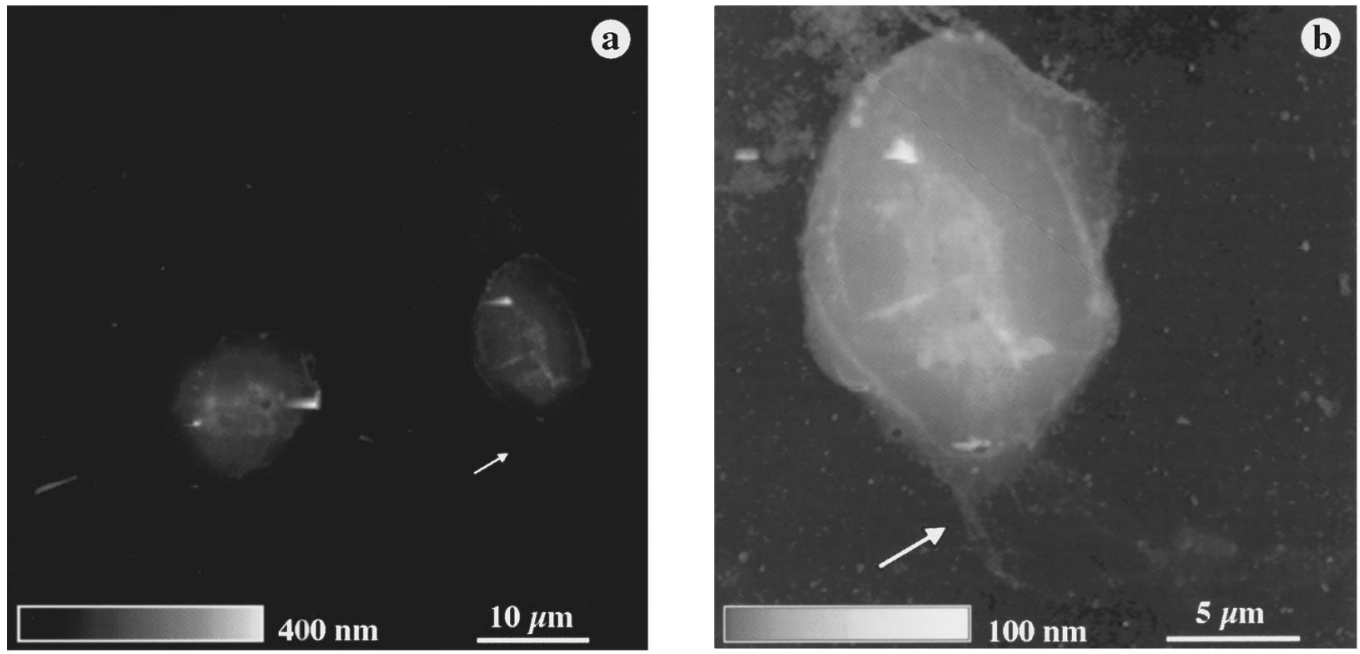

FIG. 5. SFM images at different magnifications of the chromatin plaques shown in Fig. 4. The chromatin containing structures are easily identifiable on these images by comparison to the DAPI fluorescence (Fig. 4). The extended fibers containing the hybridization signals are also revealed (arrows). For better visibility of these fibers, panel (b) representing a zoom of the structure to the right in (a) is displayed on a log scale; the line defining the plane fit was located horizontally across the fibers at the level of the arrow. The gray scales code height information, the scale bars show lateral dimensions.

chromosomes 13 and $21 .^{30}$ Thus, the FISH probe is likely to colocalize with the nucleoli, which appeared in the SFM as distinct globular domains of heights well above the remainder of the spread chromatin. Assuming a well-stretched B-DNA, the length of the labeled region of $2 \mathrm{Mbp}^{31}$ would be $\sim 700 \mu \mathrm{m}$. The observed signals were mostly round with diameters of $\sim 1-6 \mu \mathrm{m}$. This is indicative of a wellcondensed state of the labeled region.

At higher resolutions fibrous material radiating out from the plaques was more easily detected [Figs. 3(c) and 3(d)]. Filaments with different dimensions could be seen, the thinnest ones having widths of 40-65 $\mathrm{nm}$ at half-height and heights of $7-8 \mathrm{~nm}$. These dimensions are within the range of values usually measured for the $30 \mathrm{~nm}$ chromatin fiber by SFM. It is reasonable to suppose that drying the samples containing DNA wound about proteins would result in distortion of an otherwise symmetric topology, and cause flattening and widening of the structures. Nevertheless, it cannot be excluded that some of the fibers observed here were built up from several $30 \mathrm{~nm}$ filaments that stuck together during the several drying steps required for the in situ hybridization. Furthermore, some may have represented large nonhistone protein complexes attached to the $30 \mathrm{~nm}$ filaments.

Hybridization with the pUC1.77 probe yielded similar results. In Fig. 4 fluorescent images of spread chromatin hybridized with the pUC1.77 probe are shown. The fluorescent FISH signals [Fig. 4(b)] were located within the DAPI stained chromatin plaques [Fig. 4(a)]. Since large spreads of a major fraction of the nuclear material such as the one shown in Fig. 3 or that marked by the arrow in Fig. 4(a) were rare events, the probability of finding a spread region that was also labeled with FISH was small. However, such a case was detected; the arrow points at a signal spread over a length of $\sim 5 \mu \mathrm{m}$.

Usually six or eight probe-specific signals were observed in one area of DNA-specific (DAPI) fluorescence. They showed a variation in intensity; usually three or four pairs of signals with distinct intensities and sizes could be detected in one plaque. A gallery of such images is collected in Fig. 4(c). Probably the largest pair of signals represents the pericentromeric regions of intact chromosomes 1 , whereas the smaller signals could have resulted from translocations of pericentromeric chromosome 1 material to other chromosomes.

A pattern similar to that detected with DAPI staining was observed with the scanning force microscope (Fig. 5). The specificity of the DNA labeling allowed the identification of debris revealed by SFM, such as that appearing in Fig. 5(b) on the pole of the plaque opposite to the arrow.

The extended chromatin material containing the probe was also distinguishable with the SFM (arrows). An interesting finding is that hybridization signals of the pUC1.77 probe were somewhat less prominent than those produced by the L1.26 probe. Height differences of $70-150 \mathrm{~nm}$ between the signals and the surrounding chromatin were measured. The most likely explanation for this observation is that the function and physical location of the DNA sequences recognized by the probes was different. While L1.26 signals colocalized with nucleoli, the probe pUC1.77 bound to pericentromeric repeats of chromosome 1q, regions not involved in nucleolar organization.

\section{CONCLUSION}

We have demonstrated the applicability of the in situ hybridization technique for the labeling of specific DNA sequences of spread chromatin, carried out simultaneously with SFM investigations of the same specimen. The localization of DNA-containing regions with distinct topographic features in the SFM image was made possible by the DNAspecific dye, DAPI. The hybridization process allowed for the identification of specific DNA sequences, while at the same time it did not hinder the spatial resolution of the SFM; 
structures as small as 7-8 $\mathrm{nm}$ in height and $40-65 \mathrm{~nm}$ in width, possibly corresponding to the $30 \mathrm{~nm}$ chromatin filaments, were detected. A height difference between the signalcontaining and noncontaining spread chromatin material was observed, possibly owing to the deposition of large protein complexes onto condensed target DNA during the hybridization process. Our results show that FISH coupled with SFM can be used for the specific identification and lateral localization of DNA sequences in spread chromatin, enabling the ultrastructural characterization of defined genes in the SFM.

\section{ACKNOWLEDGMENTS}

The authors wish to thank Dr. Frank Speleman for the generous gift of FISH probes and Achim Schaper for valuable discussions. W.F. is supported by the German Academic Exchange Service. L.T. was supported by a short-term fellowship of the Boehringer Ingelheim Fonds. G.V. is a recipient of a postdoctoral grant of the Alexander von Humboldt Stiftung.

${ }^{1}$ R. D. Kornberg, Science 184, 868 (1974).

${ }^{2}$ M. J. Allen, X. F. Dong, T. E. O’Neill, P. Yau, S. C. Kowalczykowski, J. Gatewood, R. Balhorn, and E. M. Bradbury, Biochemistry 32, 8390 (1993).

${ }^{3}$ W. Fritzsche, A. Schaper, and T. M. Jovin, Chromosoma 103, 231 (1994).

${ }^{4}$ K. E. van Holde, Chromatin (Springer, New York, 1988).

${ }^{5}$ G. Yang, S. H. Leuba, C. Bustamante, J. Zlatanova, and K. van Holde, Nature Struct. Biol. 1, 761 (1994).

${ }^{6}$ A. S. Belmont, and K. Bruce, J. Cell Biol. 127, 287 (1994).

${ }^{7}$ T. Cremer, A. Kurz, R. Zirbel, S. Dietzel, B. Rinke, E. Schröck, M. R. Speicher, U. Mathieu, A. Jauch, P. Emmerich, H. Scherthan, T. Ried, C. Cremer, and P. Lichter, Cold Spring Harbor Symp. Quantum Biol. 58, 777 (1994).

${ }^{8}$ T. Haaf and M. Schmid, Exp. Cell Res. 192, 325 (1991).

${ }^{9}$ W. Mosgöller, A. R. Leitch, and J. K. M. Brown, Human Genetics 88, 27 (1991).
${ }^{10}$ C. Wollenberg, M. P. Kiefaber, and K. D. Zang, Human Genetics 62, 310 (1982).

${ }^{11}$ G. Binnig, C. F. Quate, and C. Gerber, Phys. Rev. Lett. 56, 930 (1986).

${ }^{12}$ A. Engel, Annu. Rev. Biophys. Biophys. Chem. 20, 79 (1991).

${ }^{13}$ C. Bustamante, D. A. Erie, and D. Keller, Cur. Opin. Struct. Biol. 4, 750 (1994).

${ }^{14}$ E. Henderson, Progr. Surf. Sci. 46, 39 (1994).

${ }^{15}$ J. H. Hoh and P. K. Hansma, Trends Cell. Biol. 2, 208 (1992).

${ }^{16}$ W. Fritzsche, J. Vesenka, and E. Henderson, Scanning Micros. 9, 729 (1995).

${ }^{17}$ S. H. Leuba, G. Yang, C. Robert, B. Samori, K. Van Holde, J. Zlatanova, and C. Bustamante, Proc. Natl. Acad. Sci. U. S. A. 91, 11621 (1994).

${ }^{18}$ L. D. Martin, J. P. Vesenka, E. Henderson, and D. L. Dobbs, Biochemistry 34, 4610 (1995).

${ }^{19}$ J. Zlatanova, S. H. Leuba, G. L. Yang, C. Bustamante, and K. Van Holde, Proc. Natl. Acad. Sci. U. S. A. 91, 5277 (1994).

${ }^{20}$ W. Fritzsche, A. Schaper, and T. M. Jovin, Scanning 17, 148 (1995).

${ }^{21}$ J. Schlammadinger, Meth. Mol. Cell. Biol. 1, 235 (1990).

${ }^{22}$ S. Joos, T. M. Fink, A. Rätsch, and P. Lichter, J. Biotechnol. 35, 135 (1994).

${ }^{23}$ C. A. J. Putman, B. G. De Grooth, J. Wiegant, A. K. Raap, K. O. Van der Werf, N. F. Van Hulst, and J. Greve, Cytometry 14, 356 (1993).

${ }^{24}$ P. Rasch, U. Wiedemann, J. Wienberg, and W. M. Heckl, Proc. Natl. Acad. Sci. U. S. A. 90, 2509 (1993).

${ }^{25}$ J. Szöllösi, S. Damjanovich, M. Balázs, P. Nagy, L. Trón, M. J. Fulwyler, and F. M. Brodsky, J. Immunol. 143, 208 (1989).

${ }^{26}$ F. Speleman, B. Van der Auwera, K. Mangelschots, M. Vercruyssen, T. Raap, J. Wiegant, M. Craen, and J. Leroy, Human Genetics 85, 569 (1990).

${ }^{27}$ H. J. Cooke and J. Hindley, Nucleic Acids Res. 6, 3177 (1979).

${ }^{28}$ P. Devilee, T. Cremer, P. Slagboom, E. Bakker, H. P. Scholl, H. D. Hager, A. F. Stevenson, C. J. Cornelisse, and P. L. Pearson, Cytogenet. Cell Genet. 41, 193 (1986).

${ }^{29}$ A. Schaper, L. Wolthaus, D. Möbius, and T. M. Jovin, Langmuir 9, 2178 (1993).

${ }^{30}$ F. S. Kaplan, J. Murray, J. E. Sylvester, I. L. Gonzalez, J. P. O'Connor, J. L. Doering, M. Muenke, B. S. Emanuel, and M. A. Zasloff, Genomics 15, 123 (1993).

${ }^{31}$ J. Meyne, E. H. Goodwin, and R. K. Moyzis, Chromosoma 103, 99 (1994). 